\title{
Ignition and Combustion in a Laminar Mixing Zone'
}

\author{
FRANK E. MARBLE ${ }^{2}$ and THOMAS C. ADAMSON, JR. ${ }^{3}$
}

California Institute of Technology, Pasadena, Calif.

\begin{abstract}
The analytic investigation of laminar combustion processes which are essentially two- or three-dimensional present some mathematical difficulties. There are, however, several examples of two-dimensional flame propagation which involve transverse velocities that are small in comparison with that in the principal direction of flow. Such examples occur in the problem of flame quenching by a cool surface, flame stabilization on a heated flat plate, combustion in laminar mixing zones, etc. In these cases the problem may be simplified by employing what is known in fluid mechanics as the boundary-layer approximation, since it was applied first by Prandtl in his treatment of the viscous flow over a flat plate. Physically it consists in recognizing that if the transverse velocity is small, the variations of flow properties along the direction of main flow are small in comparison with those in a direction normal to the main flow. The analytic deseription of the problem simplifies accordingly. The present analysis considers the ignition and combustion in the laminar mixing zone between two parallel moving gas streams. One stream consists of a cool combustible mixture, the second is hot combustion products. The two streams come into contact at a given point and a laminar mixing process follows in which the velocity distribution is modified by viscosity, and the temperature and composition distributions by conduction, diffusion, and chemical reaction. The decomposition of the combustible stream is assumed to follow first-order reaction kinetics with temperature dependence according to the Arrhenius law. For a given initial velocity, composition, and temperature distribution, the questions to be answered are: (1) Does the combustible material ignite; and (2) how far downstream of the initial contact point does the flame appear and what is the detailed process of development. Since the hot stream is of infinite extent, it is found that ignition always takes place at some point of the stream. However, when the temperature of the hot stream drops below a certain value, the distance required for ignition increases so enormously that it essentially does not occur in a physical apparatus of finite dimension. The complete development of the laminar flame front is computed using an approximation similar to the integral technique introduced by von Kármán into boundary layer theory.
\end{abstract}

\section{Nomenclature}

$A=$ activation energy, cal $/ \mathrm{mole} /{ }^{\circ} \mathrm{K}$

$c_{p}=$ specific heat at constant pressure, $c a l / g m /{ }^{\circ} \mathrm{K}$

$D=$ binary diffusion eoefficient, $\mathrm{cm}^{2} / \mathrm{sec}$

$f=$ dimensionless stream function

$P_{r}=$ Prandtl number $=c_{n \mu} / \lambda$

$Q=$ rate at which heat is added per unit volume, cal/sec/unit vol

Presented at the 8th ARS National Convention, New York, N. Y, December 3,1953 .

This investigation was carried out in part, under the fingncial sponsorship of the Ordnance Corps, U.S. Army.

"Associate Professor, the Daniel and Florence Guggenheim Jet Propulsion Center.

SResearch Engineer, Jet Propulsion Laboratory.

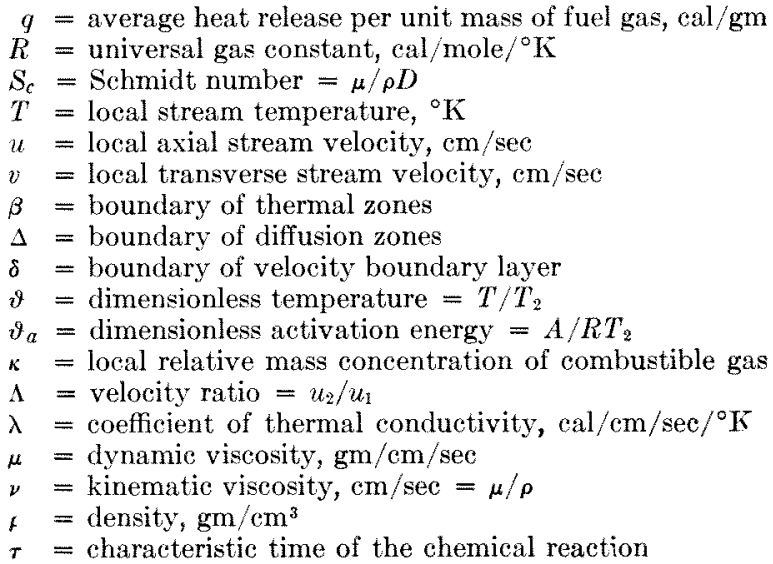

\section{Introduction}

A NALYTIC studies of combustion in flowing gaseous mixtures $(1,2,3)^{4}$ are confined, for the most part, to the plane one-dimensional flame front. The reason for this is not entirely because of the relative simplicity of this problem but also because, due to the very small thickness of the laminar combustion zone at ordinary pressures, many actual situations are well approximated by the plane flame front in spite of the fact that the actual flame front is curved.

There exist some problems, however, in which the physical situation may in no way be approximated by a plane flame front. For example, the thermal quenching of a gaseous mixture near a cool wall (4) or the thermal ignition near a heated wall essentially involve two-dimensional fields. Likewise, combustion processes in free jets and combustion under conditions of mixing between two gaseous streams require considerations beyond the one-dimensional flame theory. In many problems associated with thermal jet-propulsion systems and rocket motors, the circumstance arises where a flowing stream of combustible mixture is ignited by contact and mixing with another stream of hot gas or products of combustion. Such a process is certainly of importance near the injector of a rocket motor, in the flow and combustion of gas through the small ports of turbojet cans, and plays a definite and vital role in the flame stabilization on bluff bodies. This, in common with the other two-dimensional problems which have been mentioned, has the property that, for large stream velocities the variations of temperature, composition, and velocity are much larger in the direction normal to the stream than they are parallel to the direction of flow. In fluid mechanies, problems exhibiting this characteristic are treated by the so-called boundary layer approximation which simplifies the description of the problem by deleting certain variations along the direction of flow in comparison with those across the flow. An extension of this idea to flow with combustion allows an analytic treatment of this entire class of problems. The present treatment is restricted to laminar flow processes, in particular that of the ignition and development of a flame front in the laminar mixing zone between parallel streams of combustible gas and hot products of combustion.

i Numbers in parentheses refer to the References on page 94. 
After discussing the physical and chemical relations which apply to the problem and simplifying the equations through extension of the boundary layer concept, the initial portion of the mixing zone is investigated where the heat evolved through chemical reaction is yet small and the problem may be solved through a perturbation to the solution for mixing without combustion. Then using the integral technique introduced by von Kármán into the study of the boundary layer, the development of combustion is traced approximately from ignition through development of the plane laminar flame front.

The authors have benefited from their many opportunities of discussing this work with Professor H. S. Tsien, through whose advice the problem has become much clearer and greatly simplified. They are most grateful for his patient and continued interest in the analysis.

\section{Formulation of the Combustion Problem}

The problem of combustion in a laminar mixing zone may be formulated by considering two semi-infinite streams of gas flowing steadily parallel to the $x$-axis, one of a combustible mixture at temperature $T_{1}$ flowing with a velocity $u_{1}$, the other consisting of combustion products at a temperature $T_{2}$, less than the adiabatic flame temperature for the combustible mixture, moving with a velocity $u_{2}$. Suppose the two streams begin to mix at $x=0$. Then if no reaction takes place the process is simply one where the velocity profile develops in a well-known manner (Fig. 1) and the temperature

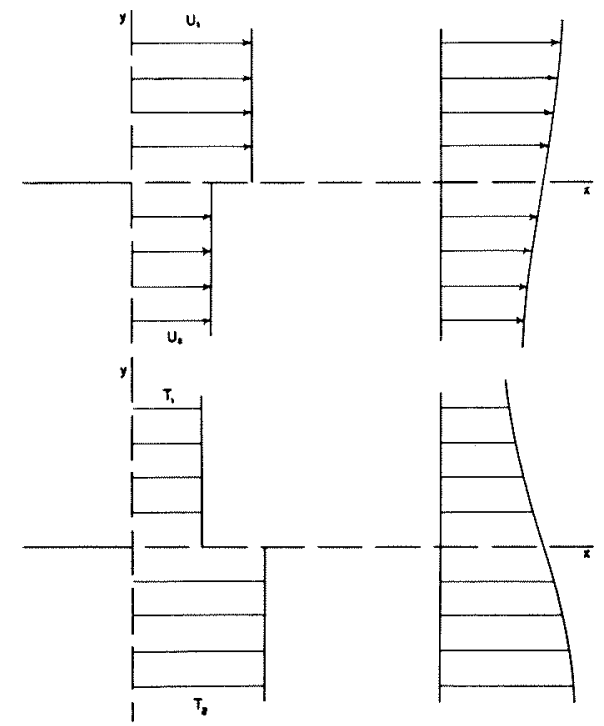

FIG. 1 VELOCITY AND TEMPERATURE PROFILES FOR A MIXING REGION WITHOUT CHEMICAT REACTION

profile grows in a somewhat similar fashion. In addition, diffusion takes place between the two streams of different gaseous composition, the combustible gas from the upper stream diffuses into the hot combustion products of the lower stream. Likewise products of combustion diffuse from the lower stream to dilute the combustible upper stream.

Now when the combustible stream reacts chemically, producing heat in the process, the change in the resulting flow is most easily observed from the development of the temperature profiles. As indicated in Fig. 2, the first noticeable modification will be the small increase in temperature caused by reaction of the gas which has diffused into the hot stream. The temperature increase takes place predominantly in the hotter part of the stream because of the extreme importance of the ambient temperature in promoting reaction. At a later stage the temperature profile is sufficiently distorted to produce a "bulge" where the local temperature exceeds that of the hot gas stream. From this point forward, the strongest

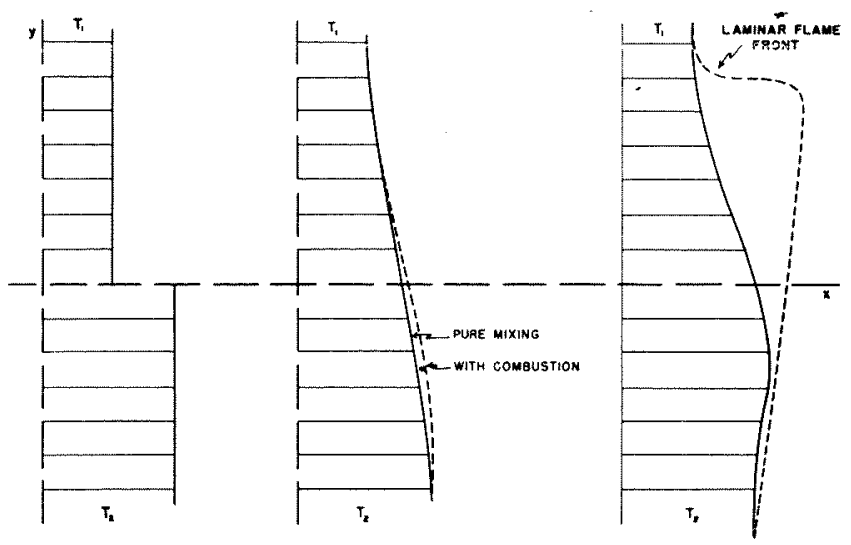

FIG. 2 TEMPERATURE PROFILES AND DEVELOPMENT OF LAMINAR FLAME IN MIXING REGION WITH CHEMICAL REACTION

reaction takes place above the maximum temperature point, due to the fact that the greater concentration of combustible gas is exposed to the higher temperatures, and consequently the point of maximum temperature moves toward the upper stream. Eventually this zone of reaction develops into a thin region resembling the laminar flame front and propagates at the appropriate speed. Since the mixing zone grows roughly as $\sqrt{x}$, it is clear that the reaction zone will move out of the laminar mixing region into the undisturbed combustible mixture and hence develops into a true plane laminar flame front. If the velocities of the two gas streams are such that laminar flame makes a very small angle with the $x$-axis, that is, if $u_{1} \gg$ laminar flame speed, then the boundary layer approximation is applicable throughout the region of flame development.

Three physical processes are involved in this problem: (A) The fluid mechanical development of the laminar mixing zone between the two streams through viscous action; (B) the development of the temperature field through conduction between the two streams and heat generated by chemical action; (C) molecular diffusion of each gaseous component into the other stream together with annihilation of combustible gas and generation of combustion products through chemical action. The first of these processes is described by the equations of motion for a viscous compressible fluid. Under the boundary layer assumptions only the dynamic equilibrium in the direction of flow need be considered. Furthermore, it is well known that due to the low value of the laminar burning speed, the pressure variations are negligible. Therefore the pressure field is uniform and, denoting by $\bar{u}, \bar{v}$ the velocity components in the $\bar{x}, \bar{y}$ directions, the equation of motion is

$$
\rho \bar{u} \frac{\partial \bar{u}}{\partial \bar{x}}+\rho \bar{v} \frac{\partial \bar{u}}{\partial \bar{y}}=\frac{\partial}{\partial \bar{y}}\left(\mu \frac{\partial \bar{u}}{\partial \bar{y}}\right)
$$

Here $\mu$ is the viscosity of the gas so that if it is assumed that the molecular weights of combustible and combustion products are the same ${ }^{5}$, the viscosity is simply proportional to $T$, the local gas temperature. Now since the gas velocities are very small in comparison with the velocity of sound, the density $\rho$ depends only upon the local gas temperature, in fact $\rho \sim 1 / T$. The two velocity components and the density are related further through the equation of continuity, expressing the conservation of mass as

$$
\frac{\partial}{\partial \bar{x}}(\rho \bar{u})+\frac{\partial}{\partial \bar{y}}(\rho \bar{v})=0 \ldots \ldots \ldots \ldots .
$$

Now since the density is a function only of the temperature,

${ }^{5}$ The three simplifying assumptions underlying this choice of gas properties are: (1) The molecular weights of both gases are equal so that the molecular weight of the mixture is independent of concentrations; (2) the gases are composed of Maxwellian molecules so that $\mu$, for example, behaves as $T$; (3) the Eucken correction for thermal conduction holds for the mixture. 
it is particularly convenient to employ the Howarth transformation which introduces a new vertical scale $(5,6)$

$$
y=\int_{0}^{\underline{y}} \frac{\rho}{\rho_{1}} d \tilde{y}
$$

where $\rho_{1}$ is the density of the combustible free stream. Calling the new coordinate system $x, y$ where $x=\bar{x}$, and new velocity components $u=\bar{u}, v=\frac{\rho}{\rho_{1}} \bar{v}+\bar{u} \int_{0}^{\bullet \dot{y}} \frac{\partial}{\partial \bar{x}}\left(\frac{\rho}{\rho_{1}}\right) d \bar{y}$, it is easy to show that the modified flow satisfies the boundary layer relations for a corresponding incompressible flow problem

$$
\begin{array}{r}
\frac{\partial u}{\partial x}+\frac{\partial v}{\partial y}=0 \ldots \\
u \frac{\partial u}{\partial x}+v \frac{\partial u}{\partial y}=\nu \frac{\partial^{2} u}{\partial y^{2}} .
\end{array}
$$

where $\nu=\mu_{1} / \rho_{1}$ is the kinematic viscosity of the combustible stream. Formally then the velocity field $u, v$ is independent of the temperature and composition fields and may be determined separately. The calculation of the real field $\bar{u}, \bar{v}$, however, requires knowledge of the temperature field to invert the transformation given by Equation [3].

The temperature field is described by the energy equation $(7,8)$ which equates the convection of thermodynamic enthalpy with the transmission by conduction and the heat generation by chemical reaction. Then assuming the value of $c_{p}$, specific heat at constant pressure, to be the same for all gas compositions and independent of temperature, the differential relation is, neglecting the pressure variation

$$
\rho \bar{u} \frac{\partial c_{p} T}{\partial \bar{x}}+\rho \bar{v} \frac{\partial c_{p} T}{\partial \bar{y}}=\frac{\partial}{\partial \bar{y}}\left(\lambda \frac{\partial T}{\partial \bar{y}}\right)+Q \ldots \ldots[6
$$

where $Q$ is the rate at which heat is being added per unit volume at a point $\vec{x}, \vec{y}$, and $\lambda$ is the thermal conductivity of the gases which will be assumed proportional to the local temperature. The heat addition rate $Q$ is determined by the heat of reaction $q,{ }^{6}$ the local mass concentration of the reacting molecules $\kappa$, and the rate at which molecules of fuel are transformed into molecules of combustion products. This rate is usually accepted by chemists to be of the form $(1 / \tau) e^{-A / R T}$, the Arrhenius factor, where $\tau$ is the characteristic time associated with a transformation, $A$ the activation energy which measures the kinetic energy a molecule must possess in order to react successfully, and $R$ the universal gas constant. For a unimolecular reaction, the rate of chemical transformation is simply the product of the local density of combustible gas with the Arrhenius factor, that is, $\kappa \rho(1 / \tau) e^{-A / R T}$. Thus the rate of heat addition by chemical reaction is simply

$$
Q=q \rho \kappa \frac{1}{\tau} e^{-A / R T}
$$

It is again convenient to apply the Howarth transformation to the energy equation so that it will be written in terms of the same quantities as the momentum equation.

$$
u \frac{\partial T}{\partial x}+v \frac{\partial T}{\partial y}=\frac{\nu}{P_{*}} \frac{\partial^{2} T}{\partial y^{2}}+\frac{q}{c_{p} \tau} \kappa e^{-A / R T} \ldots \ldots[8
$$

where $P_{r} \equiv c_{p} \mu_{1} / \lambda_{1}$ is the Prandtl number of the gases, which is constant. For nonvanishing temperature the heat evolved through chemical reaction vanishes only if the local concentration of combustible matter vanishes. This fact leads to the formal difficulty that a gas which flows from $x=-\infty$ is completely reacted unless a condition is added to disconnect the flow from $x=-\infty$. The identical problem arises in the

${ }^{6}$ In view of the assumptions concerning the constancy of molecular weight and $c_{p}, q$ is to be interpreted as the average heat release per unit mass of the fuel gas. study of the one-dimensional laminar flame and has recently been discussed by von Kármán (3). In the present work it will be treated in a manner similar to that which von Kármán has employed for one-dimensional flame propagation.

Finally the distributions of combustible gas and products of combustion must be determined through the conservation relations for the chemical species. There are two species and hence two conservation laws; however, it is clear that the two must sum to the gross continuity relation, Equation [2], and hence only one is independent. It is convenient to consider the mass concentration $\kappa$ of the combustible gas. 'The movement of this gas differs from that of the complete gas only by the diffusion velocity and the combustible mixture is destroyed locally according to the reaction rate. Then with good approximation

$$
\rho \vec{u} \frac{\partial \kappa}{\partial \bar{x}}+\rho \bar{v} \frac{\partial \kappa}{\partial \bar{y}}=\frac{\partial}{\partial \bar{y}}\left(\rho D \frac{\partial \kappa}{\partial \bar{y}}\right)-\rho \frac{\kappa}{\tau} e^{-A / R T} \ldots \ldots
$$

If the Howarth transformation is again carried out, and the Schmidt number $S_{c}=\mu / \rho D$ assumed to be constant, then the conservation of combustible material may be written

$$
u \frac{\partial \kappa}{\partial x}+v \frac{\partial \kappa}{\partial y}=\frac{\nu}{S_{c}} \frac{\partial^{2} \kappa}{\partial y^{2}}-\frac{\kappa}{\tau} e^{-A / R T} \ldots \ldots \ldots
$$

Equations [4], [5], [8], and [10] now describe the simplified combustion process which takes place in an equivalent incompressible flow. By virtue of the Howarth transformation the momentum and continuity equations may be treated independently of the temperature and composition fields and hence constitute a conventional mixing problem. The energy relation and equation for conservation of combustible material must be solved simultaneously, employing knowledge of the velocity field. Since the equations are nonlinear it is clear that solutions may be expected in only certain cases or certain regions and only approximate solutions may be obtained with any generality. Because of the importance of the ignition region, a solution will be obtained for the early portion of the mixing zone. Then, by means of an approximate treatment, the complete course of development of a laminar flame front will be examined.

\section{Initial Development of the Combustion Zone}

During the first portion of the combustion process, where the heat added by the chemical reaction is yet small, the velocity, temperature, and composition fields differ only slightly from those which would occur in the absence of chemical action. The fields without combustion may be computed easily, and consequently the variation due to combustion may be treated as a perturbation.

If the reaction were taking place at a temperature $T$ and concentration $\kappa$, the combustible material would be consumed at a rate $\kappa(1 / \tau) e^{-A / R T}$. Due to the strong influence of the Arrhenius factor, the initial reaction takes place where $T$ is nearly $T_{2}$ and the concentration $\kappa$ is very small. The actual value of $\kappa$ where the reaction takes place most rapidly may be estimated by computing the maximum value of the local reaction rate. Thus

$$
\frac{d \kappa}{d y} \frac{1}{\tau} e^{-A / R T}+\frac{\kappa}{\tau} \frac{A}{R T} \frac{1}{T} \frac{d T}{d y} e^{-A / R T}=0
$$

But the temperature distribution $\left(T_{2}-T\right) /\left(T_{2}-T_{1}\right)$ is identical with the concentration distribution $k(y)$ when the Prandtl and Schmidt numbers are equal. Then

$$
\frac{d \kappa}{d y} / \frac{d T}{d y}=\frac{-1}{T_{2}-T_{1}}
$$

and taking account of the fact that the reaction takes place where $T \approx T_{2}$, the local concentration is approximately 


$$
\kappa=\left[\frac{A}{R T_{2}}\left(1-\frac{T_{1}}{T_{2}}\right)\right]^{-1}
$$

Consequently the local rate at which combustible matter is consumed is

$$
\frac{1}{\frac{A}{R T_{2}}\left(1-T_{1} / T_{2}\right)} \frac{1}{\tau} e^{-A / R T_{2}}
$$

and the total heat release which takes place over a length $x$ is proportional to

$$
\frac{x}{u_{2} \tau} \frac{e^{-A / R T_{2}}}{\frac{A}{R T^{2}}\left(1-T_{1} / T_{2}\right)}
$$

since the reaction takes place predominantly where the gas velocity is nearly $u_{2}$. Then so long as $x$ is such that the total heat addition is small, a perturbation procedure is in order. Therefore it is appropriate to define a characteristic length associated with combustion, $l=\frac{\tau u_{2}}{R T_{2}} A\left(1-T_{1} / T_{2}\right) e^{-A / R T_{2}}$, which may be employed to make the formulation dimensionless; denote $\xi=x / l$.

Because of its important role in the early stages of combustion, the temperature $T_{2}$ of the hot stream is conveniently chosen as the characteristic temperature; denote $T / T_{2}=\vartheta$, $T_{1} / T_{2}=\vartheta_{1}$, and $A / R T_{2}=\vartheta_{a}$. The energy and diffusion equations then may be written simply as

$$
u \frac{\partial \vartheta}{\partial x}+v \frac{\partial \vartheta}{\partial y}=\frac{\nu}{P_{r}} \frac{\partial^{2} \vartheta}{\partial y^{2}}+\frac{q}{c_{p} T_{2}} \frac{\kappa}{\tau} e^{-\vartheta_{\alpha} / \vartheta} \ldots \ldots
$$

and

$$
u \frac{\partial \kappa}{\partial x}+v \frac{\partial \kappa}{\partial y}=\frac{\nu}{S_{c}} \frac{\partial^{2} \kappa}{\partial y^{2}}-{ }_{\tau}^{\kappa} e^{-\vartheta \vartheta_{a / \vartheta}}
$$

Now the momentum equation has a simple similarity solution regardless of the structure of the rest of the field. For, choosing a stream function $\psi(x, y)$ such that $u=\partial \psi / \partial y$, $v=-\partial \psi / \partial x$, it is easily shown that $\psi=\sqrt{u_{1} \nu x} f(\eta)$ where $\eta=y \sqrt{u_{1} / \nu x}$ and $f(\eta)$ satisfies the differential equation.

$$
2 f^{\prime \prime \prime}+f f^{\prime \prime}=0
$$

with boundary conditions $f^{\prime}(\infty)=1, f^{\prime}(-\infty)=\frac{u_{2}}{u_{1}}=\Lambda$, and $f^{\prime \prime}(\infty)=f^{\prime \prime}(-\infty)=0$. In the absence of chemical reaction Equations [12] and [13] also have simple solutions. The differential equations reduce directly to

$$
\frac{2}{P_{r}} \vartheta^{(0) \prime \prime}+f \vartheta^{(0) \prime}=0
$$

and

$$
\frac{2}{S_{c}} \kappa^{(0) \prime \prime}+f_{K}^{(0) \prime}=0 .
$$

with boundary conditions $\vartheta^{(0)}(\infty)=T_{1} / T_{2}=\vartheta_{1}, \vartheta^{(0)}(-\infty)=$ $1, \vartheta^{(0)^{\prime}}(\infty)=\vartheta^{(0)^{\prime}}(-\infty)=0$; and similarly $\kappa^{(0)}(\infty)=1$, $\left.\kappa^{(0)}(-\infty)=0, \kappa^{(0)^{\prime}}(\infty)=\kappa^{(0)}\right)^{\prime}(-\infty)=0$.

The presence of chemical action destroys the elementary similarity of this simple mixing problem so that the solutions $\vartheta$ and $\kappa$ cannot be functions of $\eta$ alone, but the distance $x$ must also enter. Then, for example, if $\vartheta$ depends upon both $\eta$ and $x$, Equation [12] becomes

$$
\frac{1}{P_{r}} \frac{\partial^{2} \vartheta}{\partial \eta^{2}}+\frac{f}{2} \frac{\partial \vartheta}{\partial \eta}-f^{\prime} x \frac{\partial \vartheta}{\partial x}=-\frac{q}{c_{p} T_{2}} \kappa\left(\frac{\kappa}{u_{1} \tau} e^{-\vartheta_{a} / \vartheta}\right)
$$

which reduces directly to Equation [15] when $q=0$, for then the dependence upon $x$ is unnecessary. By writing Equation [17] in the form

$$
\frac{1}{P_{r}} \frac{\partial^{2} \vartheta}{\partial \eta^{2}}+\frac{f}{2} \frac{\partial \vartheta}{\partial \eta}-f^{\prime} \xi \frac{\partial \vartheta}{\partial \xi}=-\frac{q}{c_{p} T_{2}} \Lambda \kappa \xi \vartheta_{a}\left(1-\vartheta_{1}\right) e^{-\vartheta_{a}\left(\frac{1}{\vartheta}-1\right)}
$$

then so long as

$$
\frac{x e^{-\vartheta_{a}}}{u_{2} \tau \vartheta_{a}\left(1-\vartheta_{1}\right)}=\xi \ll 1
$$

the appropriate expression for $\vartheta$ is

$$
\vartheta=\vartheta^{(0)}(\eta)+\vartheta^{(1)}(\eta) \xi+\vartheta^{(2)}(\eta) \xi^{2}+\vartheta^{(3)}(\eta) \xi^{3}+\ldots
$$

The situation is quite similar for the equations describing the conservation of combustible matter, for Equation [13] may be written

$$
\frac{1}{S_{c}} \frac{\partial^{2} \kappa}{\partial \eta^{2}}+\frac{f}{2} \frac{\partial \kappa}{\partial \eta}-f^{\prime} \xi \frac{\partial \kappa}{\partial \xi}=\Lambda \kappa \xi \vartheta_{a}(1-\vartheta) e^{-\vartheta_{a}}\left(\frac{1}{\vartheta}-1\right) .
$$

so that likewise it is appropriate to express

$$
\kappa=\kappa^{(0)}(\eta)+\kappa^{(1)}(\eta) \xi+\kappa^{(2)}(\eta) \xi^{2}+\kappa^{(3)}(\eta) \xi^{3}+\ldots
$$

It is obvious that $\vartheta^{(0)}(\eta)$ and $\kappa^{(0)}(\eta)$ satisfy Equations [15] and [16], respectively, for the pure mixing problem, while the relations to be satisfied by $\vartheta^{(n)}(\eta)$ and $\kappa^{(n)}(\eta)$ follow upon substitution into Equations [18] and [20], respectively, according to the classical perturbation technique. The firstorder perturbation gives simply

$$
\begin{aligned}
& \frac{1}{P_{r}} \frac{d^{2} \vartheta^{(1)}}{d \eta^{2}}+\frac{f}{2} \frac{d \vartheta^{(1)}}{d \eta}-f^{\prime} \vartheta^{(1)}= \\
& -\frac{q}{c_{p} T_{2}} \Lambda \kappa^{(0)} \vartheta_{a}\left(1-\vartheta_{1}\right) e^{-\vartheta_{a}\left(\frac{1}{\vartheta^{(0)}}-1\right)} \ldots \\
& \frac{1}{S_{c}} \frac{d^{2} \kappa^{(1)}}{d \eta^{2}}+\frac{f}{2} \frac{d \kappa^{(1)}}{d \eta}-f^{\prime} \kappa^{(1)}=\Lambda \kappa^{(0)} \vartheta_{a}\left(1-\vartheta_{1}\right) e^{-\vartheta_{a}\left(\frac{1}{\vartheta(0)}-1\right)}
\end{aligned}
$$

where it is necessary that these perturbations and their derivatives vanish at $\eta= \pm \infty$.

The perturbation solution may be worked out for various special cases, the simplest of which is that where the velocities of the two streams are equal, that is, where $\Lambda=1$. Then the stream function is just $f=\eta$, and it is easily demonstrated that the distributions of temperature and composition in the absence of combustion are just

$$
\begin{aligned}
& \frac{\vartheta^{(0)}(\eta)-\vartheta_{1}}{1-\vartheta_{1}}=\frac{1}{2}\left[1-\operatorname{erf}\left(\frac{1}{2} \sqrt{P_{r}} \eta\right)\right] \ldots \ldots \\
& \kappa^{(0)}(\eta)-1=\frac{1}{2}\left[1-\operatorname{erf}\left(\frac{1}{2} \sqrt{S_{\circ} \eta}\right)\right] \ldots \ldots .
\end{aligned}
$$

where $\operatorname{erf}(z)=(2 / \sqrt{\pi}) \int_{0}^{2} e^{-t^{2}} d t$. These are well-known solutions of the simple heat conduction and diffusion problems. The first-order perturbation equation for temperature distribution, Equation [22], may now be written as

$$
\begin{aligned}
\frac{1}{P_{r}} \frac{d^{2} \vartheta^{(1)}}{d \eta^{2}}+\frac{\eta}{2} \frac{d \vartheta^{(1)}}{d \eta} & -\vartheta^{(1)}= \\
& -\frac{q}{c_{p} T_{2}} \kappa^{(0)} \vartheta_{a}\left(1-\vartheta_{1}\right) e^{-\vartheta_{a}\left(\frac{1}{\vartheta^{(0)}}-1\right)}
\end{aligned}
$$

where $\vartheta^{(0)}(\eta)$ is given by Equation [24]. The homogeneous equation has two linearly independent solutions which may be written in the form

$$
\begin{aligned}
& \mathscr{K}_{1}(\eta)=\sqrt{P_{r} \eta e^{-\frac{P_{r}}{4} \eta^{2}}}+ \\
& \quad\left(\frac{1}{2}+\frac{P_{r}}{4} \eta^{2}\right) 2 \sqrt{2 \pi}\left[\operatorname{erf}\left(\frac{\sqrt{P_{r}}}{2} \eta\right)-1\right] \ldots
\end{aligned}
$$




$$
\begin{aligned}
\mathscr{K}_{2}(\eta)=\sqrt{P_{r} \eta e^{-\frac{P_{r}}{4} \eta^{2}}+} & \\
& \left(\frac{1}{2}+\frac{P_{r}}{4} \eta^{2}\right) 2 \sqrt{2 \pi}\left[\operatorname{erf}\left(\frac{\sqrt{P_{r}}}{2} \eta\right)+1\right] \ldots
\end{aligned}
$$

Then constructing the appropriate Green's function as

$G\left(\eta, \bar{\eta}_{;} \quad P_{r}\right)=\left\{\begin{array}{l}\frac{-1}{4 \sqrt{\pi P_{r}}} \mathcal{K}_{1}(\eta) \mathcal{K}_{2}(\tilde{\eta}) e^{\frac{P_{r}}{4} \bar{\eta}^{2}} ; \eta \geq \bar{\eta} \\ \frac{-1}{4 \sqrt{\pi P_{r}}} \mathfrak{K}_{1}(\bar{\eta}) \mathcal{K}_{2}(\eta) e^{\frac{P_{r}}{4} \bar{\eta}_{2}} ; \eta \leq \bar{\eta}\end{array}\right\} \ldots \ldots$

the solution of Equation [26] may be written explicitly as

$$
\begin{aligned}
\vartheta^{(1)}(\eta)=\operatorname{Pr} \frac{q}{C_{p} T_{2}} & \vartheta_{a}\left(1-\vartheta_{1}\right) \\
& \int_{-\infty}^{\infty} G\left(\eta, \bar{\eta} ; P_{r}\right) \kappa^{(0)}(\bar{\eta}) e^{-\vartheta_{a}\left(\frac{1}{\eta^{\prime}(\overline{0})}-1\right)} d \bar{\eta} .
\end{aligned}
$$

It is clear from Equation [23] that the first-order perturbation for the concentration may be obtained in a similar manner as

$\kappa^{(1)}(\eta)=-S_{c} \vartheta_{a}\left(1-\vartheta_{1}\right)$

$$
\int_{-\infty}^{\infty} G\left(\eta, \bar{\eta} ; S_{c}\right) \kappa^{(0)}(\bar{\eta}) e^{-\vartheta_{a}}\left(\frac{1}{\vartheta(\theta)}-1\right) d \bar{\eta} .
$$

where, as indicated, the Schmidt number now replaces the Prandtl number in Green's function.

This form is very suitable for numerical computation since Green's function and the rest of the integrand are easily calculable. The integration has been carried out for the particular case where $P_{r}=0.91, S_{c}=1.00, \vartheta_{1}=0.286$, $\vartheta_{a}=23.96, \frac{q}{c_{p} T_{2}}=1.29$, and $T_{1}=300^{\circ} \mathrm{K}$, where physical parameters are approximately those of the fuel azomethane. The resulting temperature profiles are given in Fig. 3 where it is clearly shown that the maximum perturbation occurs well below the center line of the mixing zone. This is due to the relatively preponderant effect of the stream temperature in increasing the reaction rate, so that although the concentration is low, the temperature is sufficiently high to react almost immediately that combustible which has diffused into this region. The temperature profiles show that very early a bulge is formed where the temperature exceeds that of the hot stream. This region continues to grow, working its way toward the combustible stream. The tendency is therefore for the initial reaction to develop into a flame since the temperatures continually increase with $x$.

The principal question is how long a time or, more realistically, how great a distance is required before the combustion takes place. The simplest significant length to measure is the value of $x$ at which the temperature profile first exhibits a vertical tangent; that is, where the local temperature has increased sufficiently to form a "bulge" in the profile. Fortunately this point may be determined analytically, for considering only the first perturbation, it is clear that

$$
\frac{\partial \vartheta}{\partial \eta} \approx \frac{d \vartheta^{(0)}}{d \eta}+\xi \frac{d \vartheta^{(1)}}{d \eta}=0
$$

Furthermore the smallest value of $\xi$ at which this condition

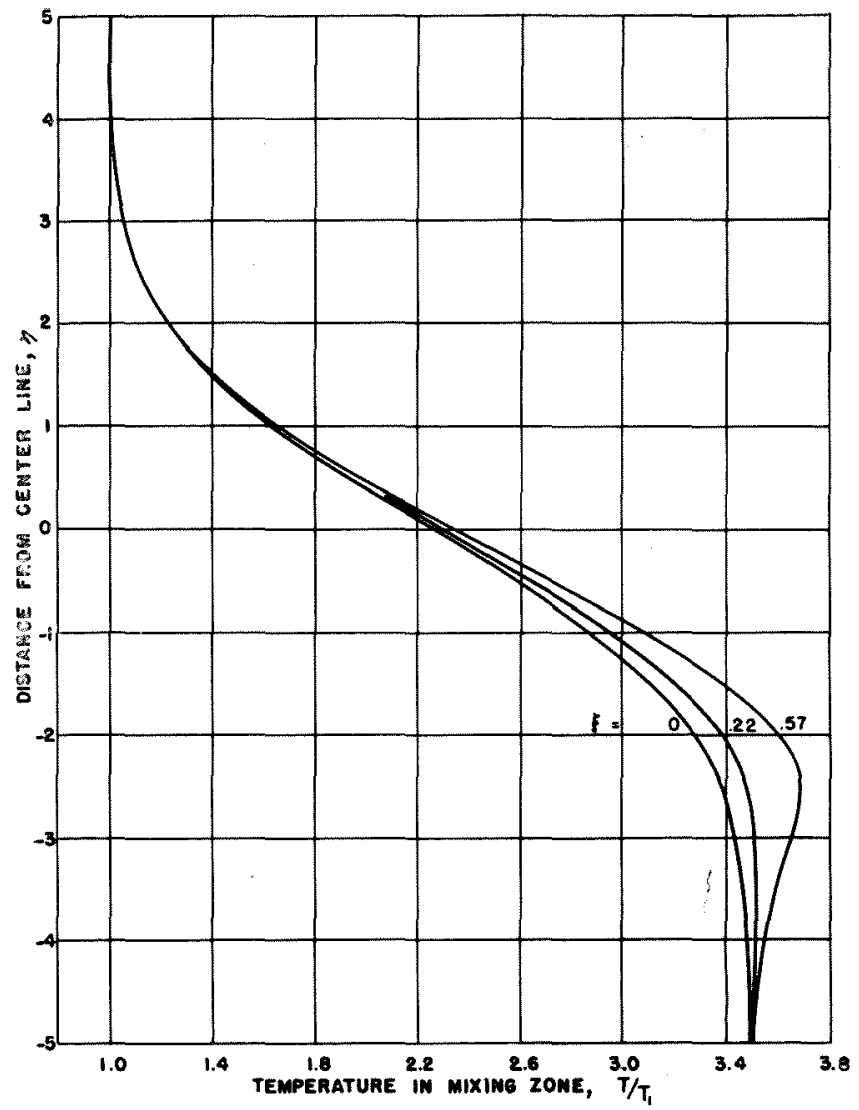

FIG. 3 DETAILED DEVELOPMENT OF TEMPERATURE PROFILES DURING THE EARLY STAGES OF REACTION

calculating from Equation [32]

$$
\frac{d^{2} \vartheta^{(0)}}{d \eta^{2}}+\xi_{i} \frac{d^{2} \vartheta^{(1)}}{d \eta^{2}}=0
$$

But now applying the differential relations for $\vartheta^{(0)}(\eta)$ and $\vartheta^{(1)}(\eta)$, given by Equation [15] and Equation [22], respectively, it follows that the value $\eta_{i}$ for which a vertical slope in the temperature is first observed is given by the simple algebraic relation

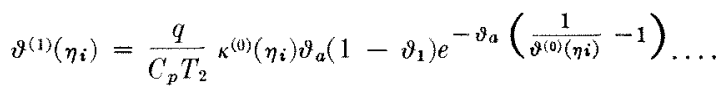

Then, according to Equation [32], the value of $\xi_{i}$ is just

$$
\xi_{i}=-\left(\frac{d \vartheta^{(0)}}{d \eta} / \frac{d \vartheta^{(1)}}{d \eta}\right)_{\eta_{i}}
$$

Now the expressions involved in Equations [33] and [34] are not simple to handle, but fortunately the values of $\eta_{t}$ which are involved may be seen, from Fig. 3 , to be $\ll-0$, so that good approximation may be made using asymptotic expansions for $\vartheta^{(1)}(\eta)$ and for the right-hand member of Equation [33]. For example, when the Prandtl number is less than unity, the asymptotic expressions for $\kappa^{(0)}(\eta)$ and $\vartheta^{(1)}(\eta)$ at large negative values of $\eta$ are

$$
\begin{aligned}
\kappa^{(0)}(\eta) \sim \frac{-1}{\sqrt{\pi} \sqrt{S_{c}}} \frac{e^{-\frac{S_{c}}{4} \eta^{2}}}{\eta}\{1 & \left.-\frac{2}{S_{c} \eta^{2}}+\ldots\right\} \quad \vartheta^{(1)}(\eta) \sim \frac{-2}{\sqrt{\pi P_{r}}} \frac{q}{c_{p} T_{2}} \vartheta_{a}\left(1-\vartheta_{1}\right)\left\{\frac{4}{S_{c}^{3 / 2}} \frac{e^{-\frac{S_{c}}{4} \eta^{2}}}{\eta^{5}}\left(1+\frac{P_{r}}{2} \eta^{2}\right) \times\right. \\
& \left(1-\left[\frac{1}{S_{c}}+\frac{1}{P_{r}}\right] \frac{12}{\eta^{2}}+\ldots\right)+\frac{e^{-\frac{P_{r}}{4} \eta^{2}}}{\eta^{3}}\left(1-\frac{12}{P_{r} \eta^{2}}+\ldots\right)\left(C_{1}-\frac{2}{\sqrt{S_{c}}} \frac{P_{r}}{S_{c}-P_{r}} e^{-\frac{\eta^{2}}{4}\left(S_{c}-P_{r}\right)}\right\} \ldots
\end{aligned}
$$

where

may be satisfied is the dimension of interest and will be denoted $\xi_{i}$. It is necessary also that $d \xi / d \eta=0$ at $\xi_{i}$, so that

$$
C_{1}=-\int_{-\infty}^{\infty} \pi_{1}(\vec{\eta}) \kappa^{(0)}(\bar{\eta}) e^{-\vartheta_{a}\left(\frac{1}{\vartheta^{(0)}}-1\right)} e^{\frac{P_{r}}{4} \bar{\eta}^{2}} d \bar{\eta}
$$




$$
\begin{array}{r}
\frac{8}{P_{r} S_{c} \eta_{i}^{4}}\left(1+\frac{P_{r}}{2} \eta_{i}{ }^{2}\right)\left(1-\left[\frac{1}{S_{c}}+\frac{1}{P_{r}}\right] \frac{12}{\eta_{i}{ }^{2}}+\ldots\right)+\frac{2 \sqrt{S_{c}}}{P_{r}} \frac{e^{\frac{\eta i^{2}}{4}\left(S_{c}-P_{r}\right)}}{\eta_{i}^{2}}\left(1-\frac{12}{P_{r} \eta_{i}^{2}}+\ldots\right)\left(C_{1}-\frac{2 P_{r} e^{-\frac{\eta i^{2}}{4}\left(S_{c}-P_{r}\right)}}{\left(S_{c}-P_{r}\right)} \sqrt{\bar{S}_{c}}\right)= \\
1-\frac{2}{S_{c} \eta_{i}^{2}}+\ldots \ldots
\end{array}
$$

After numerical solution for $\eta_{i}$, the value of $\xi_{i}$ is obtained through direct substitution into Equation [34], that is

$$
\xi_{i}=\frac{\sqrt{P_{r}}}{2} \frac{\eta_{i}^{2}}{\vartheta_{a}\left(1-\vartheta_{1}\right)}\left\{\left(C_{1}-\frac{2 P_{r} e^{\frac{-\eta i^{2}}{4}\left(S_{c}-P_{r}\right)}}{\left(S_{c}-P_{r}\right) \sqrt{S_{c}}}\right)\left(1-\frac{6}{P_{r} \eta_{i}^{2}}+\ldots\right)-\frac{8}{\eta_{i}{ }^{2}} \frac{e^{\frac{-\eta i^{2}}{4}\left(S_{c}-P_{r}\right)}}{S_{c}{ }^{3 / 2}}\left(1-\frac{12}{\eta_{i}{ }^{2}}\left[\frac{1}{S_{c}}+\frac{1}{P_{r}}\right)\right]\right\}^{-1} \ldots
$$

where the asymptotic expansions have been used for $d \vartheta^{(1)} / d \eta$. Now the principal variation of $x_{i}$ is due to the exponential term so that it is clear that as the temperature of the hot stream decreases, the detachment distance of the combustion zone increases exponentially. Using the same values of the parameters as those employed in constructing the temperature profiles, the detachment distance $x_{i}$ has been computed for a range of hot stream temperatures. Calculations incidentally showed that $\eta_{i}$ was about -6 , thereby justifying the use of asymptotic expansions in its evaluation. It appears from Fig. 4 that the value of $x_{i}$ increases enormously as the hot stream becomes cooler. Therefore it is seen that although this process of combustion in a laminar mixing zone shows no distinct blowoff, the flame detachment becomes so great for low stream temperature that it exceeds the physical dimensions of any apparatus.

Because the initial reaction occurs at such large negative values of $\eta$, it is a simple matter to extend the analysis to the

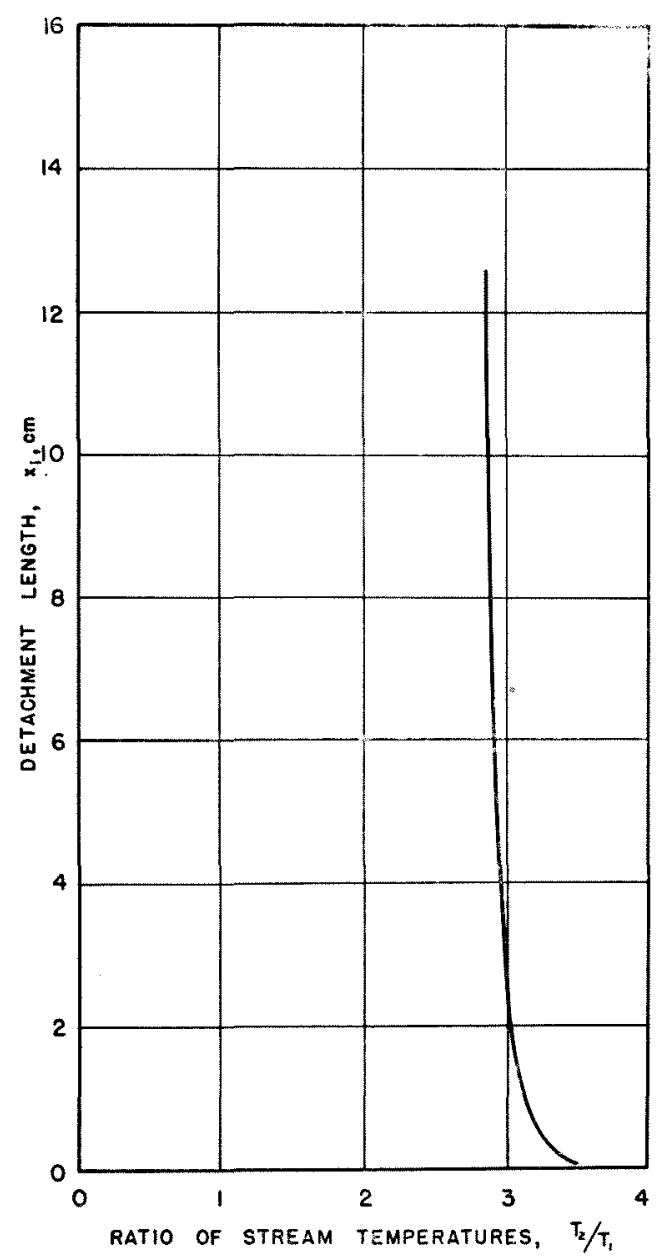

FIG. 4 VARIATION OF DETACHMENT LENGTH WITH TEMPERATURE OF THE HOT GAS STREAM case where $\Lambda \neq 1$ by employing the asymptotic expansions of the solution for the velocity field. However, this additional consideration modifies Equation [35] only to the order $\left(e^{-\frac{\eta^{2}}{4}}\right)^{2}$, and therefore Equation [37] is valid, to the present approximation, for any value of $\Lambda$ that is not too small.

\section{Development of the Laminar Flame}

The perturbation scheme employed in discussing the initial combustion zone is, of course, inappropriate for following the transition to a laminar flame front. However, since the initial region is known from the foregoing analysis, an approximate procedure is employed in extending the solution throughout the remainder of the flame zone. The technique that allows best use of the information which has so far been accumulated is the integral method introduced by von Kárman into the study of laminar and turbulent boundary layers. In this method the differential equations are integrated across the stream utilizing a descriptive knowledge of the distribution of velocity, temperature, etc. The resulting ordinary differential equations then describe the manner in which the geometric widths of assumed profiles vary along the direction of flow. Extension of von Karmán's integral method to problems of combustion simply necessitates addition of the integrated equations for species conservation to those for momentum and energy employed in the analysis of compressible boundary layer.

Since the success of the method is directly related to the reasonable choice of approximate profiles, it is necessary to be assured that profiles selected exhibit the essential physical characteristics. The velocity distribution is probably the simplest, since after the Howarth transformation it is independent of temperature or composition variations. The two fluid streams are initially separate and it is convenient to describe them by separate integral relations. The streamline which divides the two fluids is not straight but deflects during the mixing process. This deflection is negligibly small, however, and the division will be taken at the line $y=0$; the velocity $u_{0}$ is constant along this streamline (9). Now let $\delta_{1}$ represent the extent of the mixing zone into the combustible mixture and $-\delta_{2}$ the extent into the hot combustion products. The momentum integral for the upper stream is then obtained by integrating the momentum equation, Equation [5], over the range $0 \leqslant y \leqslant \delta_{1}$, in the conventional manner (10), to obtain

$$
\frac{d}{d x} \int_{0}^{\delta_{1}(x)} \frac{u}{u_{1}}\left(1-\frac{u}{u_{1}}\right) d y=\nu \frac{\partial}{\partial y}\left(\frac{u}{u_{1}}\right)_{y=0} \ldots \ldots
$$

Similarly in the lower fluid region

$$
\frac{d}{d x} \int_{-\delta_{2}(x)}^{0} \frac{u}{u_{1}}\left(\Lambda-\frac{u}{u_{1}}\right) d y=-\nu \frac{\partial}{\partial y}\left(\frac{u}{u_{1}}\right)_{y=0}
$$

The results of these two relations must match at the dividing line so that the viscous shear be continuous. Since all fluid properties are continuous at this point, 


$$
\frac{\partial}{\partial y}\left(\frac{u}{u_{1}}\right)_{y=0+}=\frac{\partial}{\partial y}\left(\frac{u}{u_{1}}\right)_{y=0-} .
$$

The energy integrals are established through Equation [12]; suppose the extent of the thermal layer above the $x$-axis is $\beta_{1}(x)$, while that below is $-\beta_{2}(x)$. It is convenient to make a division between the "upper" and "lower" streams along a line $\beta_{0}(x)$ above which the reaction rate may be neglected. If the temperature of the hot stream is not too great, this may be taken as the isotherm having a temperature $\vartheta=\vartheta_{0}$ corresponding to that of the dividing streamline $(y=0)$ in the absence of combustion. Then integrating over the region $y>\beta_{0}(x)$ gives

$$
\begin{aligned}
& \frac{d}{d x} \int_{\beta_{0}(x)}^{\beta_{1}(x)} \frac{u}{u_{1}}\left(\vartheta_{1}-\vartheta\right) d y-\left(\vartheta_{0}-\vartheta_{1}\right) \\
& \frac{d}{d x} \int_{0}^{\beta_{0}(x)} \frac{u}{u_{1}} d y=\left.\frac{\nu}{P_{r} U_{1}} \frac{\partial y}{\partial y}\right|_{y=\beta_{0}(x)}
\end{aligned}
$$

and for the region $y<\beta_{0}(x)$

$$
\begin{aligned}
\frac{d}{d x} \int_{-\beta_{2}(x)}^{\beta_{0}(x)} \frac{u}{u_{1}}(1-\vartheta) d y- & \left(1-\vartheta_{0}\right) \\
\frac{d}{d x} \int_{0}^{\beta_{0}(x)} \frac{u}{u_{1}} d y= & -\left.\frac{\nu}{P_{r} u_{1}} \frac{\partial \vartheta}{\partial y}\right|_{y=\beta_{0}(x)}- \\
& \frac{q}{c_{p} T_{2} u_{1}} \tau \int_{-\beta_{2}(x)}^{\beta_{0}(x)} \kappa e^{-\vartheta_{a} / \vartheta} d y
\end{aligned}
$$

For these equations the matching between the two regions expresses the physical fact that the heat conduction is continuous across $\beta_{0}(x)$, that is

$$
\left.\frac{\partial \vartheta}{\partial y}\right|_{\beta_{0}+}=\left.\frac{\partial \vartheta}{\partial y}\right|_{\beta_{0}-}
$$

Integration of the concentration relation [13], presents the additional complication that the concentration $\kappa\left(\beta_{0}\right)$ is not constant. Integration above the line $\beta_{0}(x)$ leads to the relation

$$
\begin{aligned}
& \frac{d}{d x} \int_{\beta_{0}(x)}^{\Delta_{1}(x)} \frac{u}{u_{1}}(1-\kappa) d y+\left(1-\kappa\left(\beta_{0}\right)\right) \\
& \frac{d}{d x} \int_{0}^{\beta_{0}(x)} \frac{u}{u_{1}} d y=\left.\frac{\nu}{S_{c} u_{1}} \frac{\partial \kappa}{\partial y}\right|_{\beta_{0}+} \ldots
\end{aligned}
$$

and below the line $\beta_{0}(x)$

$$
\begin{aligned}
\frac{d}{d x} \int_{-\Delta_{2}(x)}^{\beta_{0}(x)} \frac{u}{u_{1}} \kappa d y-\kappa\left(\beta_{0}\right) \int_{0}^{\beta_{0}(x)} \frac{u}{u_{1}} d y= \\
\left.\quad \frac{\nu}{S_{c} u_{1}} \frac{\partial \kappa}{\partial y}\right|_{\beta_{0}-}-\frac{1}{\tau u_{1}} \int_{-\Delta_{2}(x)}^{\beta_{0}(x)} \kappa e^{-v_{\alpha} / \vartheta} d y \ldots
\end{aligned}
$$

where $\Delta_{1}(x)$ and $-\Delta_{2}(x)$ are the thicknesses of the diffusion zone in the upper and lower streams respectively. The continuity of diffusive transport of combustible matter at $\beta_{0}(x)$ gives the matching relation

$$
\left.\frac{\partial \kappa}{\partial y}\right|_{\beta_{0}+}=\left.\frac{\partial \kappa}{\partial y}\right|_{\beta_{0}-} \ldots
$$

The perturbation analysis of the previous section disclosed the fact that the temperature profiles have a different character accordingly as the value of $x$ is less than or greater than $x_{i}$ where a temperature maximum first appears. To illustrate the manner in which this fact exhibits itself in the present approximate treatment, consider the situation where the Prandtl number is less than the Schmidt number so that the diffusion of combustible matter into the lower stream takes place more slowly than the increase of the thermal boundary layer thickness, that is, $\beta_{2}>\Delta_{2}$ for the initial reaction zone. Clearly then, the reaction takes place most rapidly at the highest temperature and consequently near the boundary $\Delta_{2}$ of the diffusion zone. In spite of the low concentration of combustible material, the temperature increases in this region causing a reduction in thickness $\beta_{2}$ of the lower thermal layer. In order that a combustion wave develop, it is necessary that the reaction eventually take place at a high temperature so that the thermal layer must decrease in thickness at a rate exceeding that at which the diffusion layer decreases due to consumption of combustible. Consequently the diffusion layer must exceed the thickness of the thermal layer after sufficient distance has been covered. As soon as this occurs, there exists a zone of reaction in the region where the temperature of the gas already is equal to $T_{2}$ and hence the local temperature will exceed $T_{2}$. This corresponds to the first appearance of a maximum in the temperature profile which was observed in the perturbation calculation, and consequently it is clear that $\Delta_{2}=\beta_{2}$ at a distance $x_{i}$ downstream. Furthermore, a bifurcation occurs in the thermal layer thickness at $x_{i}$, and for $x>x_{i}$; one branch lies above the maximum temperature, the other at the edge of the lower thermal zone, below the maximum. It is only the lower branch which possesses a particular physical significance. Therefore, although an approximation to the velocity and concentration profiles may be obtained which is valid over the whole mixing region, the temperature profile must be approximated differently accordingly as the point in question lies upstream or downstream of the distance where a maximum is first obtained in the temperature profile.

It is both convenient and reasonably accurate to represent the various profiles by appropriate portions of trigonometric functions. For example, Lock (9) has employed this approximation in one method of treating the viscous layer between two parallel streams of gas. For the velocity profile the distribution will be

$$
\begin{aligned}
\frac{u}{u_{1}} & =\frac{u_{0}}{u_{1}}+\left(1-\frac{u_{0}}{u_{1}}\right) \sin \frac{\pi}{2} \frac{y}{\delta_{1}} ; y>0 \\
& =\frac{u_{0}}{u_{1}}+\left(\frac{u_{2}}{u_{1}}-\frac{u_{0}}{u_{1}}\right) \sin \frac{\pi}{2}\left(\frac{y}{-\delta_{2}}\right) ; y<0 \ldots
\end{aligned}
$$

The concentration distribution is also relatively simple except that it must be written with respect to the concentration $\kappa\left(\beta_{0}\right)$ and with respect to the limit $\beta_{0}(x)$ of the combustion zone. Therefore

$$
\begin{aligned}
\kappa & =\kappa\left(\beta_{0}\right)+\left(1-\kappa\left(\beta_{0}\right)\right) \sin \frac{\pi}{2}\left(\frac{y-\beta_{0}}{\Delta_{1}-\beta_{0}}\right) ; y>\beta_{0} \\
& =\kappa\left(\beta_{0}\right)-\kappa\left(\beta_{0}\right) \sin \frac{\pi}{2}\left(\frac{y-\beta_{0}}{-\Delta_{2}-\beta_{2}}\right) ; y<\beta_{0} \ldots \ldots
\end{aligned}
$$

Now for the region $x<x_{i}$ the temperature profile may also be approximated in this elementary manner

$$
\begin{aligned}
\vartheta & =\vartheta_{0}+\left(\vartheta_{1}-\vartheta_{0}\right) \sin \frac{\pi}{2}\left(\frac{y-\beta_{0}}{\beta_{1}-\beta_{0}}\right) ; y>\beta_{0} \\
& =\vartheta_{0}+\left(1-\vartheta_{0}\right) \sin \frac{\pi}{2}\left(\frac{y-\beta_{0}}{-\beta_{2}-\beta_{0}}\right) ; y<\beta_{0} \ldots
\end{aligned}
$$

but for $x>x_{i}$ it must be possible for the profile to show a maximum temperature. To accomplish this in a relatively simple manner it is sufficiently realistic to assume that for any value of $x$, the maximum temperature occurs at the lower boundary of the diffusion zone, that is, where $y=-\Delta_{2}(x)$. The maximum temperature is then just $\vartheta\left(-\Delta_{2}\right)$ and the temperature profiles are

$$
\begin{aligned}
& \vartheta=\vartheta_{0}+\left(\vartheta_{1}-\vartheta_{0}\right) \sin \frac{\pi}{2}\left(\frac{y-\beta_{0}}{\beta_{1}-\beta_{0}}\right) ; y>\beta_{0} \\
& =\vartheta_{0}+\left(\vartheta\left(-\Delta_{2}\right)-\vartheta_{0}\right) \sin \frac{\pi}{2}\left(\frac{y-\beta_{0}}{-\Delta_{2}-\beta_{0}}\right) ;-\Delta_{2}<y<\beta_{0} \\
& =\frac{\vartheta\left(-\Delta_{2}\right)+1}{2}-\frac{\vartheta\left(-\Delta_{2}\right)-1}{2} \cos \frac{\pi}{2}\left(\frac{-y-\beta_{2}}{-\Delta_{2}-\beta_{2}}\right) \\
& -\beta_{2}<y<-\Delta_{2} \ldots[50]
\end{aligned}
$$


Substitution of these profiles into the preceding six integral equations yields six simultaneous ordinary differential equations for the functions $\delta_{1}(x), \delta_{2}(x), \Delta_{1}(x), \Delta_{2}(x), \beta_{1}(x)$, and $\beta_{2}(x)$. As an illustration it is instructive to use the same example employed in the perturbation analysis, that is, where $u_{1}=u_{2} \equiv u$. Then only four functions are involved, $\delta_{1}(x)$ and $\delta_{2}(x)$ being absent since no shearing stresses are present. Furthermore the temperature $\vartheta_{0}$ is just $\left(\vartheta_{1}+1\right) / 2$, since it is that value which would occur at the interface in the absence of combustion. From the trigonometric relations themselves it is easily shown that $\beta_{0}(x)=1 / 2\left(\beta_{1}-\beta_{2}\right)$ while the concentration along $\beta_{0}(x)$ is $\kappa\left(\beta_{0}\right)=\left(\beta_{0}+\Delta_{2}\right) /\left(\Delta_{1}+\Delta_{2}\right)$. Upon evaluation of the integrals in Equations [41], [42], [44], and [45], the following differential equations are obtained for the region $x<x_{i}$, after some simplification

$$
\begin{array}{r}
\frac{d}{d x}\left(\Delta_{1}+\Delta_{2}\right)=\frac{\pi^{2}}{2(\pi-2)} \frac{\nu}{S_{\mathrm{c} u} u}\left(\frac{1}{\kappa\left(\beta_{0}\right)\left(1-\kappa\left(\beta_{0}\right)\right)}\right)\left(\frac{1}{\Delta_{1}+\Delta_{2}}\right)- \\
\frac{4}{\pi-2} \frac{\kappa\left(\beta_{0}\right)\left(\Delta_{1}+\Delta_{2}\right) I}{\left(1-\vartheta_{1}\right) u r e^{-\vartheta_{a}}}
\end{array}
$$$$
\frac{d}{d x}\left(\kappa\left(\beta_{0}\right)\right)=\frac{\pi^{2}}{4(\pi-2)} \frac{\nu}{S_{c} u} \frac{1-2 \kappa\left(\beta_{0}\right)}{\kappa\left(\beta_{0}\right)\left(1-\kappa\left(\beta_{0}\right)\right)} \frac{1}{\left(\Delta_{1}+\Delta_{2}\right)^{2}}+
$$$$
\frac{2 \kappa\left(\beta_{0}\right)}{\pi-2}\left[\frac{\frac{q}{c_{p} T_{2}}+1-\vartheta_{1}}{1-\vartheta_{1}} \kappa\left(\beta_{0}\right)-1\right] \frac{I}{\left(1-\vartheta_{1}\right) u \tau e^{\vartheta} a}
$$

$$
\begin{aligned}
\frac{d}{d x}\left(\beta_{1}-\beta_{0}\right)= & \frac{\pi^{2}}{2(\pi-2)} \frac{\nu}{P_{r} u} \frac{1}{\left(\beta_{1}-\beta_{0}\right)}- \\
& \frac{4}{\pi-2} \frac{q}{c_{p} T_{2}\left(1-\vartheta_{1}\right)} \kappa^{2}\left(\beta_{0}\right)\left(\Delta_{1}+\Delta_{2}\right) \frac{I}{\left(1-\vartheta_{1}\right) u \tau e^{\vartheta_{a}}}
\end{aligned}
$$

$\frac{d \beta_{0}}{d x}=\frac{4}{\pi} \frac{q}{c_{p} T_{2}\left(1-\vartheta_{1}\right)} \kappa^{2}\left(\beta_{0}\right)\left(\Delta_{1}+\Delta_{2}\right) \frac{I}{\left(1-\vartheta_{1}\right) u \tau e^{\vartheta_{a}}}$.

where $I$ is a definite integral over the reaction zone given by

$$
I=\int_{\vartheta_{0}}^{1} \sqrt{\frac{1-\frac{\vartheta-\vartheta_{0}}{1-\vartheta_{0}}}{1+\frac{\vartheta-\vartheta_{0}}{1-\vartheta_{0}}}} e^{-\vartheta_{a}\left(\frac{1}{\vartheta}-1\right)} d \vartheta \ldots
$$

Now since a numerical integration is obviously called for, it is necessary to investigate the behavior of the solutions to Equations [51] near the origin where integration must begin. The expansions are naturally in powers of $\xi$ and may be computed as

$$
\begin{aligned}
\frac{\Delta_{1}+\Delta_{2}}{\sqrt{\frac{\nu x}{u}}} & =\frac{2 \pi}{\sqrt{S_{c}(\pi-2)}}\left(1-\frac{I \vartheta_{a}}{\pi-2} \xi+\ldots\right) \\
\kappa\left(\beta_{0}\right) & =\frac{1}{2}+\left(\frac{q}{c_{p} T_{2}\left(1-\vartheta_{1}\right)}-1\right) \frac{I \vartheta_{a}}{3(\pi-2)} \xi+\ldots \\
\frac{\beta_{0}}{\sqrt{\frac{\nu x}{u}}} & =\frac{2 \pi}{\sqrt{S_{c}(\pi-2)}} \frac{q}{c_{p} T_{2}\left(1-\vartheta_{1}\right)} \frac{2 I \vartheta_{a}}{3 \pi} \xi+\ldots \\
\frac{\beta_{1}-\beta_{0}}{\sqrt{\frac{v x}{u}}} & =\frac{\pi}{\sqrt{P_{r}(\pi-2)}}\left(1-\sqrt{\frac{P_{r}}{S_{c}}} \frac{I \vartheta_{a}}{\pi-2} \times\right. \\
\sqrt{\frac{\pi}{u}} & \left.\frac{q}{c_{p} T_{2}\left(1-\vartheta_{1}\right)} \xi+\ldots\right) \ldots
\end{aligned}
$$

Actually these expressions give a fairly accurate idea of the behavior of the mixing and reaction zones for $x<x_{i}$. For example, the value of $x_{i}$ itself, determined by the condition $\Delta_{2}\left(x_{i}\right)=\beta_{2}\left(x_{i}\right)$, may be calculated from these results by noting that since $\Delta_{2}+\beta_{2}=\kappa\left(\beta_{0}\right)\left(\Delta_{1}+\Delta_{2}\right)$ and $\beta_{2}+\beta_{0}=\beta_{1}-\beta_{0}$, the value of $x_{i}$ satisfies the relation $\beta_{1}\left(x_{i}\right)-\beta_{2}\left(x_{i}\right)=\kappa\left(\beta_{0}\right)$ $\left[\Delta_{1}\left(x_{i}\right)+\Delta_{2}\left(x_{i}\right)\right]$. Then from Equations [53] it follows directly that

$$
\xi_{1}=\frac{\sqrt{\frac{S_{c}}{P_{r}}-1}}{\vartheta_{a}\left[\frac{q}{c_{p} T_{2}\left(1-\vartheta_{1}\right)}-1\right]}\left(\frac{3(\pi-2)}{5 I}\right)
$$

The complete calculation is shown in Fig. 5 for this region for numerical values corresponding to azomethane which have been employed earlier. The results are essentially the same as those found by the perturbation analysis.

The differential relations which describe the development of combustion for $x>x_{i}$ are more involved than those describing the initial region due both to the more complex temperature profiles and to the initial conditions which must be used at $x=x_{i}$ where integration is started. Furthermore, there are now five of these differential equations and they may be written as

$$
\begin{array}{r}
\frac{d}{d x}\left(\Delta_{1}+\Delta_{2}\right)=\frac{\pi_{2}}{2(\pi-2)} \frac{\nu}{S_{c} u} \frac{1}{\kappa\left(\beta_{0}\right)\left(1-\kappa\left(\beta_{0}\right)\right)} \frac{1}{\Delta_{1}+\Delta_{2}}- \\
\frac{4}{\pi-2}\left(\beta_{1}-\beta_{0}\right) \frac{I\left(\Delta_{2}, \beta_{0}\right)}{u \tau\left(1-\vartheta_{1}\right) e^{\vartheta} a}
\end{array}
$$

$$
\begin{aligned}
& \frac{d}{d x}\left(\beta_{1}-\beta_{0}\right)=\frac{4-\pi}{4 \kappa\left(\beta_{0}\right) \frac{\Delta_{1}+\Delta_{2}}{\beta_{1}-\beta_{0}}+\pi} \times \\
& \quad\left\{\frac{\pi^{2}}{2(\pi-2)} \frac{\nu}{S_{c} u \kappa\left(\beta_{0}\right)\left(1-\kappa\left(\beta_{0}\right)\right)\left(\Delta_{1}+\Delta_{2}\right)} \times\right. \\
& {\left[1+2 \frac{S_{c}}{P_{r}-\pi} \frac{\pi}{4-\pi}\left(1-\kappa\left(\beta_{0}\right)\right)\right]+\frac{\pi^{3}}{2(\pi-2)(4-\pi)} \frac{\nu}{P_{r} u} \times} \\
& \quad \frac{1}{\beta_{1}-\beta_{0}}-\frac{4}{\pi-2} \frac{I\left(\Delta_{2}, \beta_{0}\right)}{u \tau\left(1-\vartheta_{1}\right) e^{\vartheta_{a}}}\left(\beta_{1}-\beta_{0}\right) \times \\
& \left.\quad\left[1+\kappa\left(\beta_{0}\right)+\frac{4(\pi-2)}{4-\pi} \frac{q}{c_{p} T_{2}\left(1-\vartheta_{1}\right)} \frac{\beta_{1}-\beta_{0}}{\Delta_{1}+\Delta_{2}}\right]\right\}
\end{aligned}
$$$$
\frac{d \kappa\left(\beta_{0}\right)}{d x}=\frac{1}{2\left(\Delta_{1}+\Delta_{2}\right)}\left\{\left(1-\kappa\left(\beta_{0}\right)\right) \frac{d}{d x}\left(\Delta_{1}+\Delta_{2}\right)-\frac{d}{d x}\left(\beta_{1}-\beta_{0}\right)+\right.
$$$$
\frac{\pi^{2}}{2(\pi-2)} \frac{\nu}{S_{c} u} \frac{1}{\left(\Delta_{1}+\Delta_{2}\right)\left(1-\kappa\left(\beta_{0}\right)\right)} \times
$$$$
\left.\left[\frac{S_{c}}{P_{r}} \frac{\left(\Delta_{1}+\Delta_{2}\right)\left(1-\kappa\left(\beta_{0}\right)\right)}{\left(\beta_{1}-\beta_{0}\right)}-1\right]\right\}
$$

$$
\frac{d \beta_{0}}{d x}=\frac{\pi}{2} \frac{\nu}{P_{r} u} \frac{1}{\left(\beta_{1}-\beta_{0}\right)}-\frac{\pi-2}{\pi} \frac{d}{d x}\left(\beta_{1}-\beta_{0}\right)
$$$$
\frac{d}{d x}\left[\left(\beta_{2}-\Delta_{2}\right)\left(\frac{\kappa\left(\beta_{0}\right)\left(\Delta_{1}+\Delta_{2}\right)}{\beta_{1}-\beta_{0}}-1\right)\right]+
$$$$
2\left[\frac{\kappa\left(\beta_{0}\right)\left(\Delta_{1}+\Delta_{2}\right)}{\beta_{1}-\beta_{0}}-1\right]\left[\frac{d}{d x}\left(\kappa\left(\beta_{0}\right)\left(\Delta_{1}+\Delta_{2}\right)\right)-\frac{d}{d x}\left(\beta_{1}-\beta_{0}\right)\right]
$$

where now

$$
I\left(\Delta_{2}, \beta_{0}\right)=\int_{\vartheta_{0}}^{\vartheta\left(-\Delta_{2}\right)} \sqrt{\frac{1-\frac{\vartheta-\vartheta_{0}}{\vartheta\left(-\Delta_{2}\right)-\vartheta_{0}}}{1+\frac{\vartheta-\vartheta_{0}}{\vartheta\left(-\Delta_{2}\right)-\vartheta_{0}}}} e^{-\vartheta_{a}}\left(\frac{1}{\vartheta}-1\right) d \vartheta
$$

The development of the combustion zone into a laminar flame front was calculated by numerical integration of the differential Equations [51] and [55]. The resulting value of the gas temperature at $y=-\Delta_{2}$ is shown in Fig. 6 from the value $x=x_{i}$ on to the point where a normal flame front appears. The exponential increase of the maximum temperature is shown as the gas moves away from the point of initial mixing. 


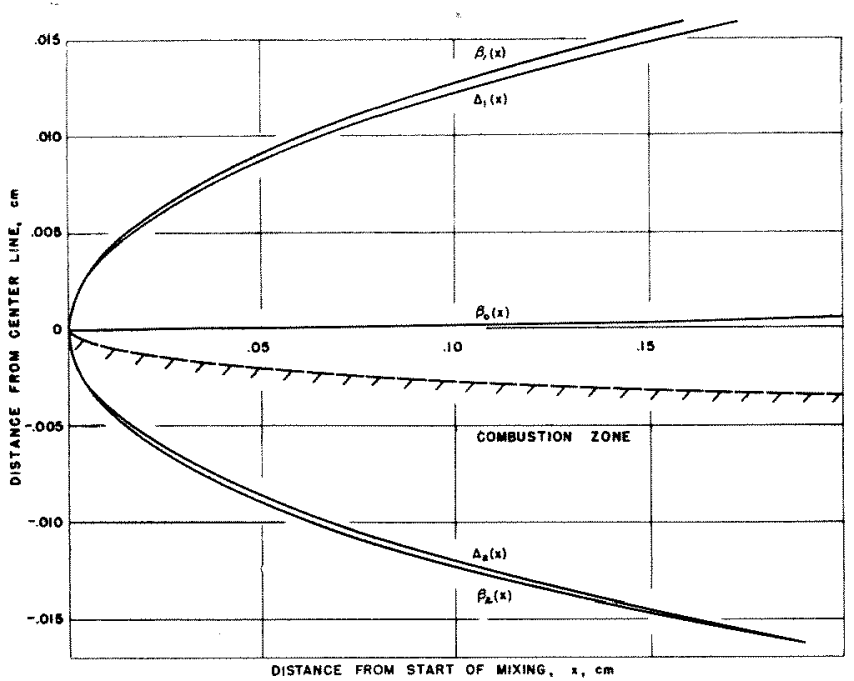

FIG. 5 GROWTH OF THICKNESSES OF TEMPERATURE AND COMPOSITION LAYERS IN THE INITIAL REACTION ZONE

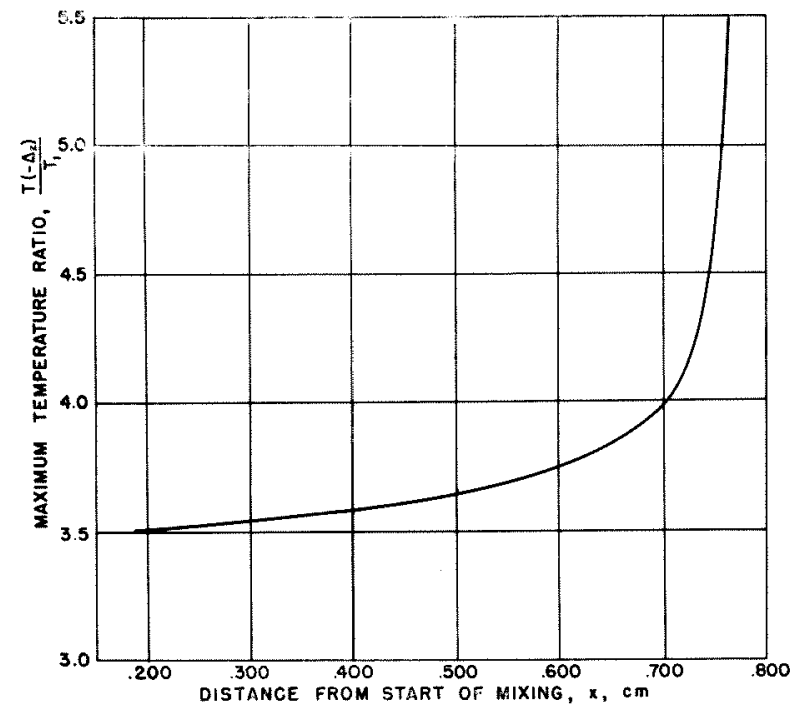

FIG. 6 INCREASE OF MAXIMUM GAS TEMPERATURE IN DIRECTION OF FLOW DURING THE LATER STAGES OF FLAME DEVELOPMENT

The detailed progress of the various regions and zones are traced in Fig. 7, in which the vertical scale is exaggerated, and demonstrates well the relatively sudden appearance of the laminar flame propagating into combustible mixture. This is indicated by the rapid convergence of the lines representing different temperatures and hence the appearance of strong temperature gradients. Also of interest is the observation (both from Fig. 6 and from Fig. 7) that the small perturbation analysis is probably adequate to describe the major portion of the reaction zone preceding the appearance of a laminar

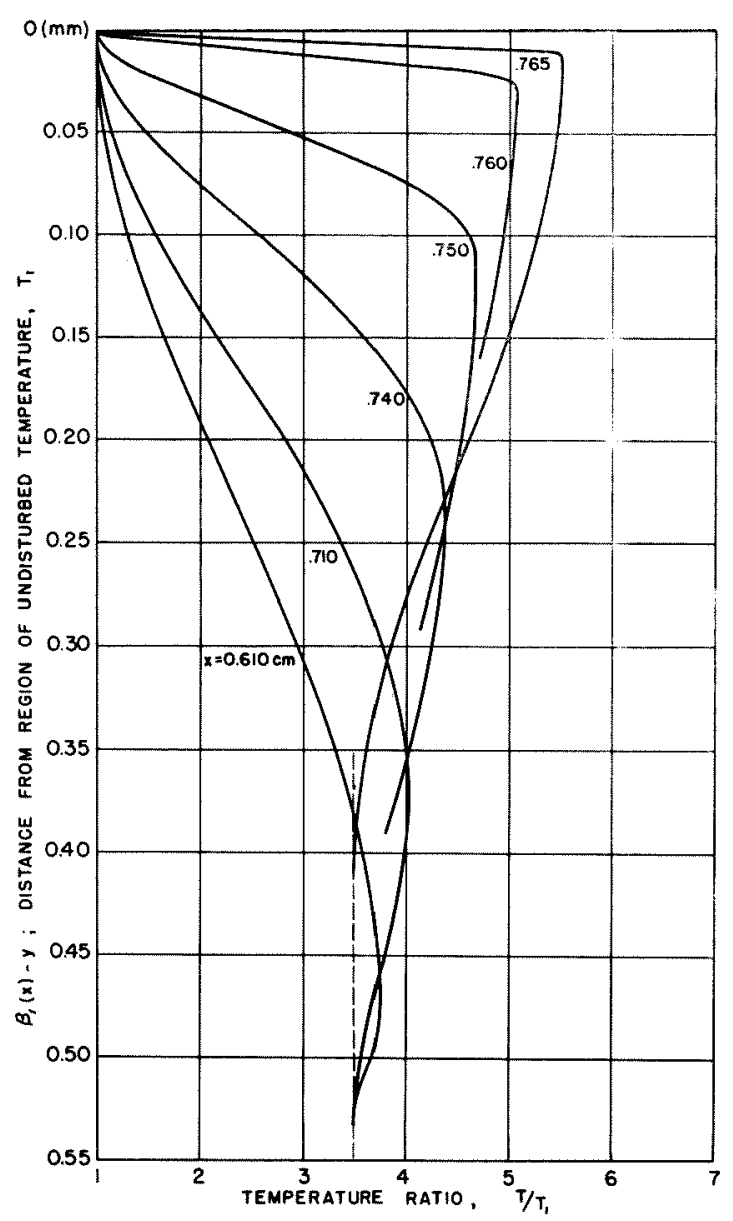

FIG. 8 TEMPERATURE PROFILES LEADING TO FINAL LAMINAR FLAME

flame front. The temperature distribution profiles which occur near the end of the ignition zone, plotted in Fig. 8, show the rapidity with which the temperature gradient steepens to the laminar flame front. It is particularly striking that the major steepening of the gradient takes place in the last 10 per cent of the reaction zone.

\section{Concluding Remarks}

Through application of a boundary layer type of analysis to the problem of ignition and combustion in the mixing zone between parallel streams of combustible gas and combustion products, some of the essential features have been deduced with relative ease. In particular, although the stream of combustible gas eventually ignites, the distance (or time) required is excessive where the temperature of the hot stream is too low. Then the flame develops so far downstream that it is essentially "blown off" any finite apparatus.

In a broader sense the analysis shows that a new class of combustion problems is open to investigation through extension of the usual boundary layer concepts. In addition

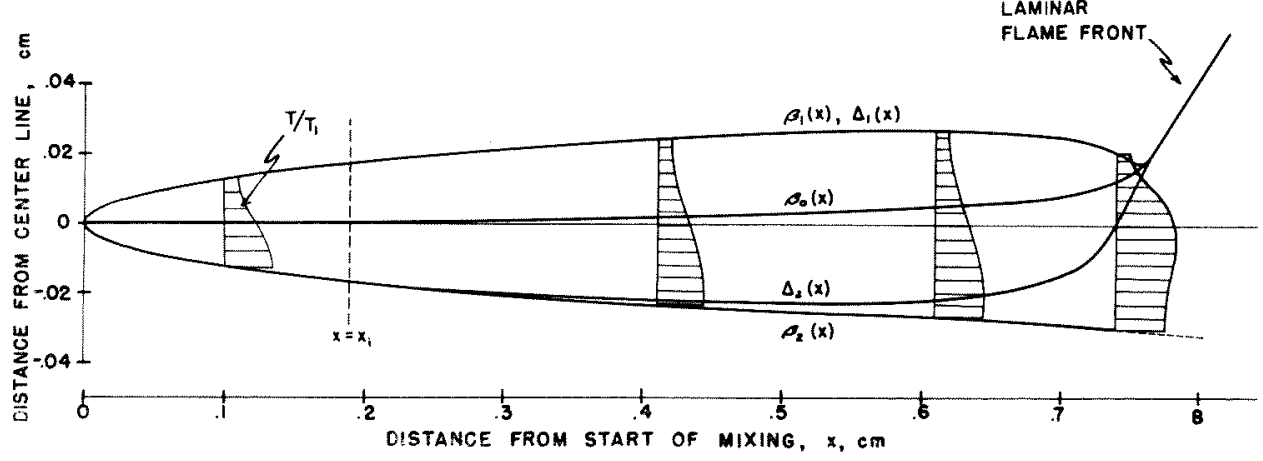

FIG. 7 GROWTH OF THICKNESSES OF TEMPERATURE AND COMPOSITION LAYERS DURING THE ENTIRE COURSE OF FLAME DEVELOPMENT 
to the present one, this class includes such important problems as the theory of thermal quenching near a cool wall, the ignition and flame stabilization on a heated surface from which the boundary layer is unseparated, the erosive burning of solid propellent grains, and a great many others. There is no essential difficulty in proceeding to problems with axial symmetry and it seems quite possible that the process may be extended to include cases of turbulent mixing. Thus the theory of the plane laminar flame together with the description of such boundary layer regions as may be treated by the methods just described, seem to provide an adequate description of a relatively large group of problems which involve steady constant pressure deflagration.

\section{References}

1 "Recherches Expérimentelles et Théoretiques sur la Combustion des Mélanges Gazeuses Explosifs," by E. Mallard and H. Le Chatelier, Ann. mines, Paris, vol. 8, series 4, 1883, p. 274.

2 "The Structure of the Reaction Zone in a Flame," by S. F. Boys and J. Corner, Proceedings of the Royal Society of London, vol. 197, series A, 1949, p. 90.

3 "The Thermal Theory of Constant Pressure Deflagration," by Theodore von Kármán and Gregorio Millan. Biezeno Anniversary Volume on Applied Mechanics, N. V. De Technische Uitgeverij H. Stam, Haarlem, Antwerpen, Djakarta, 1953, pp. 59-69.

4 "Thermal Theory of a Laminar Flame Front Near a Cold Wall," by Theodore von Kármán and Gregorio Millan. Fourth International Symposium on Combustion, Williams \& Wilkins, Baltimore, 1953.

5 "Laminar Boundary Layers in Compressible Fluids," by A. Dorodnitzyn, C. R. Academy of Sciences, URSS, vol. 34, no. 8, 1952, pp. 213-219.

6 "Concerning the Effect of Compressibility on Laminar Boundary Layers and Their Separation," by P. L. Howarth, Proceedings of the Royal Society of London, vol. 194, series A, No. A1036, 1948, pp. 16-42.

7 "The Mathematical Theory of Non-Uniform Gases," by S. Chapman and T. G. Cowling, University Press, Cambridge, 2nd ed., 1942, pp. 218-258.

8 "The Kinetic Theory of Gases," by J. O. Hirschfelder, C. F. Curtiss, R. B. Bird, and E. L. Spotz, C. F. 1500A, University of Wisconsin, 1952, p. 88.

9 "The Velocity Distribution in the Laminar Boundary Layer Between Parallel Streams," by R. C. Lock, Quart. J. Mechanics and A pplied Mathematics, vol. IV, pt. 1, 1951,pp. 42-63. 10 "Modern Developments in Fluid Mechanics," by S. Goldstein, Clarendon Press, Oxford, 1st ed., 1938, pp.131-134. 